\title{
Isolated choanal and gut atresias: pathogenetic role of serine protease inhibitor type 2 (SPINT2) gene mutations unlikely
}

\author{
Christian Niederwanger' ${ }^{1}$ Silvia Lechner ${ }^{2}$, Lisa König ${ }^{3}$, Andreas R. Janecke ${ }^{3}$, Claus Pototschnig4, \\ Beatrice Häussler ${ }^{5}$, Sabine Scholl-Bürgi ${ }^{3}$, Thomas Müller ${ }^{3}$ and Peter Heinz-Erian ${ }^{3^{*}}$ (D)
}

\begin{abstract}
Background: Choanal (CA) and gastrointestinal atresias (GA) are an important feature of syndromic congenital sodium diarrhea (SCSD), a disorder recently associated with mutations in the gene for serine protease inhibitor type 2 (SPINT2). It is, however, not known whether isolated non-syndromic CA and GA themselves might result from SPINT2 mutations.
\end{abstract}

Methods: We performed a prospective cohort study to investigate 19 CA and/or GA patients without diarrhea ("nonsCSD") for potential SCSD characteristic clinical features and SPINT2 mutations.

Results: We found a heterozygous SPINT2 splice mutation (c.593-1G>A), previously demonstrated in SCSD in homozygous form, in only 1 of the 19 patients of the "non-sCSD" cohort. This patient presented with isolated anal atresia and borderline low laboratory parameters of sodium balance. In the remaining 18 non-sCSD CA/GA patients investigated, SPINT2 sequence analysis and clinical markers of sodium homeostasis were normal. None of the 188 healthy controls tested in a regional Tyrolean population harbored the c.593-1G $>$ A mutation, which is also not listed in the ExAc and gnomAD databases.

Conclusions: The finding of only one heterozygous SPINT2 mutation in 19 patients with isolated CA/GA was not statistically significant. Therefore, SPINT2 mutations are an unlikely cause of non-SCSD atresia.

Trial registration ISRCTN73824458. Retrospectively registered 28 September 2014

Keywords: Isolated atresia, Gut, Choanae, Serine protease inhibitor type 2, SPINT2 splice mutation

\section{Background}

Atresias of the choanae (CA) [1] and the gut (GA) have been shown to be due to a large variety of pathomechanisms [2-6]. One disorder also commonly exhibiting CA and/or GA is autosomal recessive syndromic congenital sodium diarrhea (sCSD) [7]. The phenotype of sCSD involves voluminous watery stools and enormous fecal losses of sodium, leading to hyponatremia and metabolic acidosis. The disease is caused by homozygous and compound heterozygous mutations in the SPINT2 gene [7].

\footnotetext{
*Correspondence: Peter.Heinz-Erian@i-med.ac.at

${ }^{3}$ Department of Pediatrics I, Medical University of Innsbruck, Anichstrasse 35, 6020 Innsbruck, Austria

Full list of author information is available at the end of the article
}

SPINT2 encodes for Kunitz-type serine protease inhibitor type two, also reported as placental bikunin [8] and hepatocyte growth factor activator type 2 [9], which has been shown to inhibit a number of serine proteases such as matriptase, prostasin and furin [10-12]. The latter have been postulated to regulate the activity of the epithelial sodium channel $(\mathrm{ENaC})$ which allows uptake of sodium by many cell types, including the intestinal epithelium [12]. SPINT2 mutations in SCSD patients may interfere with the normal process of regulated proteolytic activity and thus lead to defective function of $\mathrm{ENaC}$. This could result in enhanced diarrheal sodium loss via the gut epithelium [7] as well as frequent occurrence of atretic malformations in SCSD, although the pathomechanism 
is currently incompletely understood. We investigated patients with isolated forms of CA and GA, who did not suffer from sodium-losing diarrhea (non-sCSD atresia), with regard to SPINT2 mutations and clinical picture, and compared the results with those from SCSD patients.

\section{Patients and methods}

The study was approved by the ethics committee of the Medical University of Innsbruck. 84 patients diagnosed with CA and/or GA between January 1, 1980 and December 31, 2011 were identified from the patient registries of the University Hospitals of Innsbruck and invited for a clinical examination. 19 patients ( 9 males, 10 females, aged 2-40 years) were included in the study after giving written informed consent. A careful history and physical examination by an ear nose and throat (ENT) specialist, a pediatric surgeon and a pediatrician was performed. Type and localization of the respective atresia was determined from previous history and physical examination data, diagnostic imaging results and surgery reports. Laboratory workup consisted of electrolytes, blood gases, osmolality and creatinine in venous plasma as well as of electrolytes, osmolality and creatinine in urine. The concentrations of sodium and creatinine in plasma and urine were used for the calculation of the fractional excretion of sodium $(\mathrm{FENa})$ using the formula $\mathrm{FENa}=$ urine $\mathrm{Na} \times$ plasma creatinine $\times 100 /($ plasma $\mathrm{Na} \times$ urine creatinine). Concentrations of $\mathrm{uNa}$ (urine sodium) and uCrea (urine creatinine) were used for the calculation of the urine sodium to urine creatinine $(\mathrm{uNa} / \mathrm{uCrea})$ ratio. Furthermore, stool samples were analyzed for electrolytes, $\mathrm{pH}$ and osmolality.

SPINT2 mutational analysis was performed as described previously [7]: briefly, genomic DNA was prepared from leukocytes using a robot (GenoM 48, Qiagen, Vienna, Austria). The complete coding region of the SPINT2 gene and all exon-intron-boundaries were amplified using intronic primers. The 190-504-bp amplicons were sequenced and the sequencing reactions analyzed on an ABI 3100 DNA sequencer. A panel of 188 DNA samples from anonymous healthy subjects was investigated for the presence of sequence variants [7]. Clinical and mutational analysis data of the 19 non-CSD atresia patients were compared with published results from 16 patients with sCSD [7]. Data of clinical parameters are presented as mean \pm SD. The statistical significance of differences between both patient groups was determined using the two-tailed Student's $t$ test. Allele frequencies for SPINT2 variants were taken from the ExAC and gnomAD databases.

\section{Results}

Among non-sCSD CA or GA patients, no homozygous or compound heterozygous mutations were found in the present study (Table 1 upper panel). However, two heterozygous SPINT2 nucleotide alterations were identified in exon seven of two non-sCSD patients with anal atresia. c.598G $>C$ het, demonstrated in patient \#10 (Table 1), was found to be a common polymorphism with an allele frequency of $2.8 \%$ in 61.000 individuals (ExAC database). c.593-1G $>$ A het, detected in a second nonsCSD atresia patient (patient \#14, Table 1 upper panel) of our study is a splice mutation that had been identified in homozygous state in five previously reported Austrian sCSD patients [7]. This mutation affects the canonical acceptor splice site in intron 6 of the SPINT2 gene. It is not listed in public databases of population-based exome and genome data, i.e., neither in ExAC (http:// exac.broadinstitute.org/gene/ENSG00000167642) nor in gnomAD (http://gnomad.broadinstitute.org/gene/ ENSG00000167642). It emerged that all five homozygous patients and the heterozygous patient \#14 of the present study originated from the same rural area, suggesting that patient \#14 of the non-sCSD group most likely was a heterozygous sCSD carrier.

The individual clinical data of the non-sCSD atresia and SCSD patients are summarized in Table 1. The average age of the non-sCSD atresia group was $139 \pm 101$ months (range 25-480 months) and thus significantly higher $(p<0.05)$ than the age of the sCSD patients (60 \pm 70 months, range 4-192 months). The gestational age of non-sCSD atresia patients was also significantly higher than that of the SCSD group $(38.8 \pm 1.9$ vs. $36.7 \pm 2.6$ weeks, $p<0.05$ ). Birth weight which was $3136 \pm 554 \mathrm{~g}$ for non-sCSD atresia patients and $2900 \pm 448 \mathrm{~g}$ for the sCSD group was not significantly different $(p=0.19)$. Diarrhea was not reported in any of the non-CSD atresia subjects, while it was the predominant feature in all sCSD patients. Moreover, average values of laboratory indicators of sodium balance (fecal $\mathrm{Na}$, plasma $\mathrm{Na}$, urinary $\mathrm{Na}, \mathrm{FE}_{\mathrm{Na}}$ and $\mathrm{uNa} / \mathrm{uCrea}$ ratio) and of metabolic acidosis were normal in the non-sCSD atresia group and were thus significantly different from the SCSD cohort where these parameters were generally pathological (Table 2). There was also no diarrhea reported in the non-sCSD atresia patient with the heterozygous splice mutation c.593-1G>A (patient \#14). However, the FENa and $\mathrm{uNa} / \mathrm{uCrea}$ in that patient showed borderline low values for these two sodium balance parameters and also a rather low uNa concentration (Table 1). We speculate that these results reflect an effect of the c.593-1G>A carrier state on sodium status, because we had previously found low urinary sodium concentrations in two obligate heterozygous sCSD parents [13]. This resembles findings in other autosomal recessive disorders, where clinically healthy patients have been shown to exhibit pathological laboratory markers as in, e.g., alpha-1-antitrypsin deficiency $[14,15]$. Apart from patient \#14, however, 


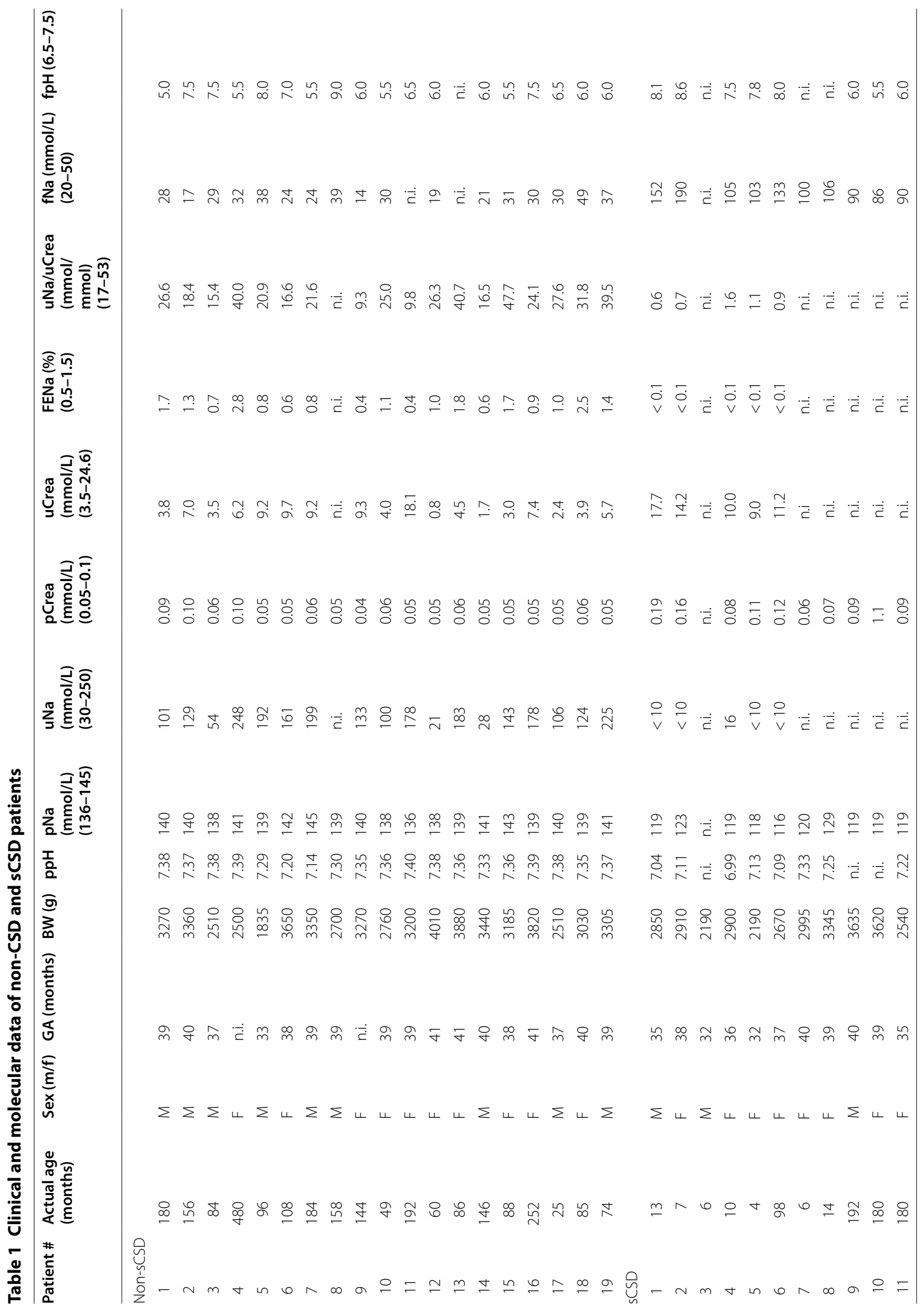




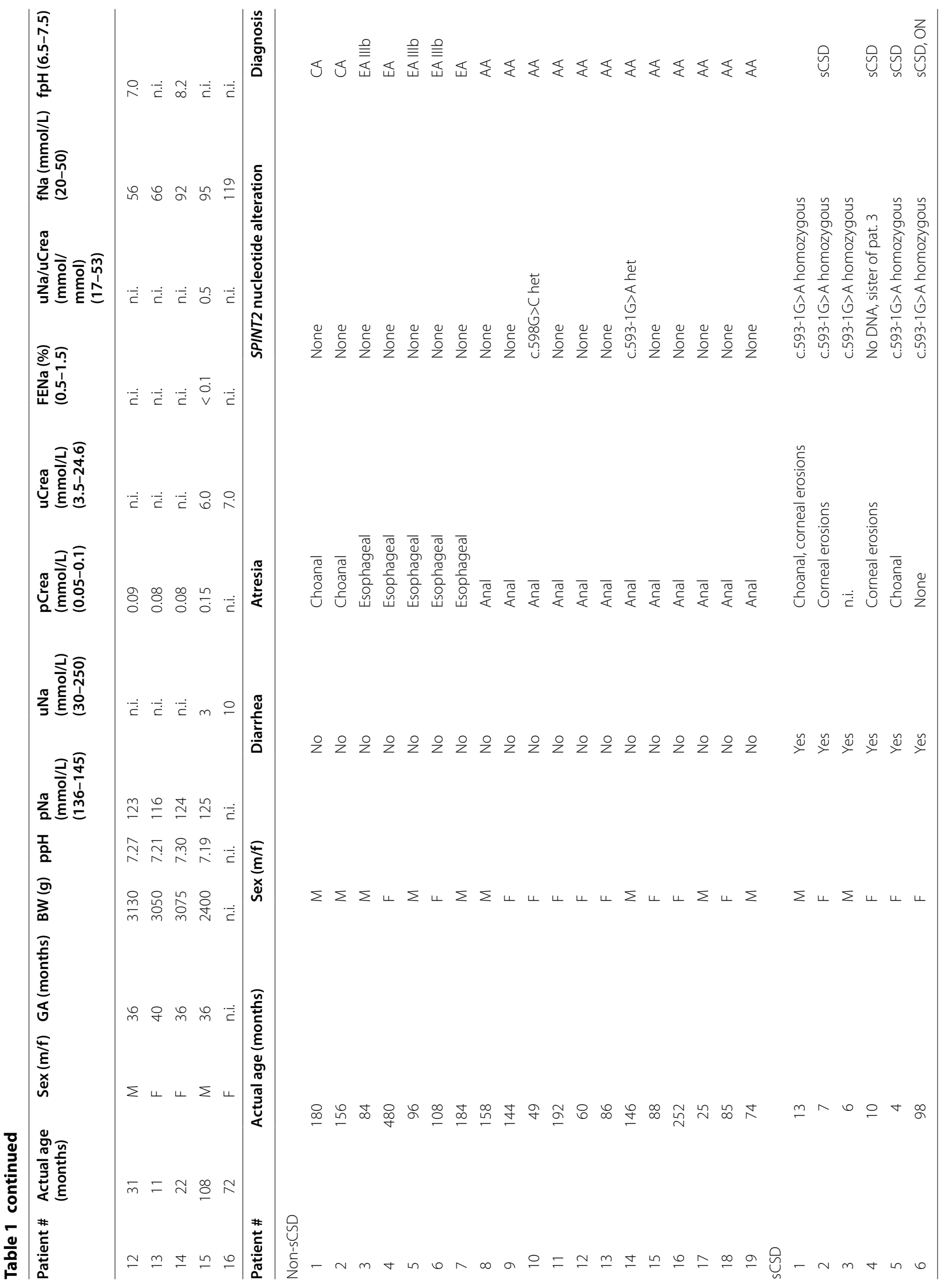




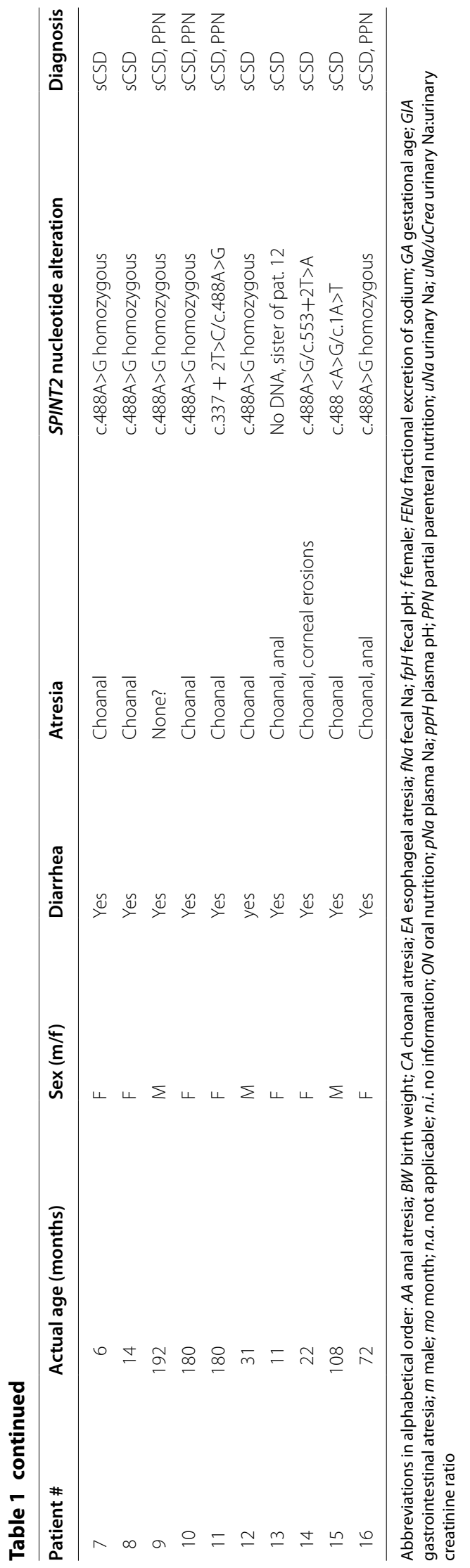




$\begin{aligned} & \text { Table } 2 \text { Clinical } \\
& \text { between non-sCSD and sCSD patients }\end{aligned}$
\begin{tabular}{lccc} 
parameters & reflecting & differences \\
\hline Parameter & non-sCSD & sCSD & p value \\
\hline Actual age (months) & $139 \pm 101$ & $60 \pm 70$ & $<0.05$ \\
Gestational age (weeks) & $39 \pm 2$ & $37 \pm 3$ & $<0.05$ \\
Birth weight (g) & $3136 \pm 554$ & $2900 \pm 448$ & n.s. \\
Plasma Na (mmol/L) & $140 \pm 2$ & $120 \pm 4$ & $<0.001$ \\
Plasma pH & $7.34 \pm 0.07$ & $7.18 \pm 0.11$ & $<0.001$ \\
Urinary Na (mmol/L) & $139 \pm 64$ & $10 \pm 4$ & $<0.001$ \\
FE & $1.2 \pm 0.7$ & $0.1 \pm 0.0$ & $<0.001$ \\
uNa/uCrea (mmol/mmol) & $25.0 \pm 11.1$ & $0.9 \pm 0.4$ & $<0.001$ \\
Fecal Na (mmol/L) & $29 \pm 9$ & $106 \pm 33.2$ & $<0.001$ \\
Fecal pH & $6.5 \pm 1.1$ & $7.3 \pm 1.1$ & n.s. \\
\hline
\end{tabular}

Abbreviations in alphabetical order: $F E_{N a}$ fractional excretion of sodium; mo month; $n$.s. not significant; $u \mathrm{Na} / u \mathrm{Crea}$ urinary $\mathrm{Na}$ /urinary creatinine ratio; $w k$ week

the results in all other 18 non-sCSD patients were normal. Besides the findings in the non-sCSD cohort, the absence of the c.593-1G>A mutation among 188 ethnically matched controls argued against a role for SPINT2 in isolated CA/GA.

\section{Discussion}

It has been previously shown that mutations in SPINT2 are associated with SCSD [7], a disease featuring a sodium-losing diarrhea, recurrent corneal erosions and frequently atretic malformations of the choanae (CA) and the gastrointestinal tract (GA). Furthermore, intestinal biopsies have shown tufts, consisting of focal crowds of enterocytes within the epithelium [16]. Mutational analysis of SPINT2 had previously identified five distinct homozygous or compound heterozygous mutations [7] in each of the investigated SCSD patients (Table 1, lower panel). Our study was undertaken to investigate whether the SPINT2 mutations identified in SCSD would also be present in patients with non-syndromic forms of isolated atresias, who did not have diarrhea (non-sCSD).

No homozygous or compound heterozygous SPINT2 mutations were identified in isolated non-sCSD atresia subjects. However, in the non-sCSD patient \#14 we found a heterozygous splice mutation, c.593-1G>A, which is identical to a SPINT2 mutation shown previously in five Austrian sCSD patients [7]. This patient also had borderline laboratory values (Table 1) for sodium balance, indicating potential sodium depletion. This suggests that carriers of the c.593-1G>A SPINT2 mutation might be recognizable by testing for phenotypical laboratory markers of sodium status (e.g., $\mathrm{FENa}, \mathrm{uNa}$ /uCrea).

Regulation of the cell surface serine protease matriptase by the SPINT2-encoded hepatocyte growth factor inhibitor 2 (HAI2) has been shown to be essential for organogenesis [17]. Furthermore, loss of SPINT2 protein has been implicated in severe clefting of the embryonic ectoderm in mice [18]. This also raises the possibility of an influence of SPINT2 on the development of $\mathrm{CA}$ and GA during prenatal organ formation. Another mechanism by which SPINT2 mutations may lead to atretic malformations could be related to the function of the epithelial sodium channel $(\mathrm{ENaC})$. This channel has been shown to be regulated by alterations of the serine protease-protease inhibitor balance which may, if defective, disturb the volume of the epithelial surface liquid layer [19]. Such a mechanism has been shown to be the case for the airway epithelium of cystic fibrosis (CF) patients in which hyperabsorption of sodium (and fluid) causes increased viscosity of the epithelial surface liquid. An analogous pathomechanism of epithelial fluid-electrolyte imbalance due to malfunction of the cystic fibrosis transmembrane regulator (CFTR) could be imagined to cause adhesion of epithelial layers inducing the commonly encountered aplasia of the vas deferens in CF [20].

\section{Conclusion}

Our study aimed at investigating whether a similar mechanism of epithelial adhesion potentially related to SPINT2 mutations could be responsible for the development of isolated CA and/or GA. However, our finding that no homozygous mutations were identified in the non-sCSD group argues against the possibility of SPINT2 mutations being a cause of isolated CA or GA.

\section{Authors' contributions}

CM organized the study, supervised the patient management, collected the data and wrote the first draft of the manuscript. SL performed mutation analysis of SPINT2. LK assisted in data collection and patient management. AJ devised and supervised genetic studies and wrote the genetic part of the paper. CP performed ENT investigations. BH provided surgical investigations. SSB provided laboratory investigations. TM provided general support of the study and proof-read the manuscript. PH-E devised and organized the study, wrote the final manuscript and submitted the manuscript as the corresponding author. All authors read and approved the final manuscript.

\section{Author details \\ ${ }^{1}$ Department of Pediatrics III, Medical University of Innsbruck, Anichstrasse 35, 6020 Innsbruck, Austria. ${ }^{2}$ VAMED, 1230 Vienna, Austria. ${ }^{3}$ Department of Pediatrics I, Medical University of Innsbruck, Anichstrasse 35, 6020 Inns- bruck, Austria. ${ }^{4}$ Department of Ear, Nose and Throat Diseases, Medical University of Innsbruck, Anichstrasse 35, 6020 Innsbruck, Austria. ${ }^{5}$ Department of General Surgery, Pediatric Surgery Unit, Medical University of Innsbruck, Anichstrasse 35, 6020 Innsbruck, Austria.}

\section{Acknowledgements}

Not applicable.

\section{Competing interests}

The authors declare that they have no competing interests.

Availability of data and materials

Trial registration: ISRCTN ISRCTN73824458. Registered 28 September 2014 Peter.heinz-erian@tirol-kliniken.at 


\section{Ethics approval and consent to participate}

The study was approved by the ethics committee of the Medical University of Innsbruck (UN3987 session 288/4.10).

\section{Funding}

This study was supported by grants from the Jubiläumsfonds der Österreichischen Nationalbank Grant \#14496 and the Tiroler Wissenschaftsfonds Grant \#UNI-404/1286 to Andreas Janecke.

\section{Publisher's Note}

Springer Nature remains neutral with regard to jurisdictional claims in published maps and institutional affiliations.

Received: 15 October 2017 Accepted: 23 February 2018

Published online: 02 March 2018

\section{References}

1. Kancherla V, Romitti PA, Sun L, et al. Descriptive and risk factor analysis for choanal atresia: The National Birth Defects Prevention Study. Eur J Med Genet. 2014:57(5):220-9.

2. Spitz L. Oesophageal atresia. Orphanet J Rare Dis. 2007;2:24.

3. Bednarczyk D, Sasiadek MM, Smigiel R. Chromosome aberrations and gene mutations in patients with esophageal atresia. J Pediatr Gastroenterol Nutr. 2013:57(6):688-93.

4. Dalla Vecchia LK, Grosfeld JL, West KW, et al. Intestinal atresia and stenosis: a 25 year experience with 277 cases. Arch Surg. 1998;133(5):490-6.

5. Boles ET Jr, Vassy LE, Ralston M. Atresia of the colon. J Pediatr Surg. 1976;11(1):69-75.

6. Spouge D, Baird PA. Imperforate anus in 700,000 consecutive liveborn newborns. Am J Med Genet. 1986;25(Suppl. 2):151-61.

7. Heinz-Erian P, Müller T, Krabichler B, et al. Mutations in SPINT2 cause a syndromic form of congenital sodium diarrhea. Am J Hum Genet. 2009:84(2):188-96.

8. Delaria KA, Muller DK, Marlor CW, et al. Characterization of placental bikunin, a novel human serine protease inhibitor. J Biol Chem. 1997;272(18):12209-14
9. Kawaguchi T, Qin L, Shimomura T, et al. Purification and cloning of hepatocyte growth factor activator inhibitor type 2, a Kunitz-type serine protease inhibitor. J Biol Chem. 1997;272(44):27558-64.

10. Planes $C$, Leyvraz C, Uchida T, et al. In vitro and in vivo regulation of transepithelial lung alveolar sodium transport by serine proteases. Am J Physiol Lung Cell Mol Physiol. 2005;288(6):L1099-109.

11. Donaldson SH, Hirsh A, Li DC, et al. Regulation of the epithelial sodium channel by serine proteases in human airways. J Biol Chem. 2002:277(10):8338-45.

12. Szabo R, Bugge TH. Membrane-anchored serine proteases in vertebral cell and developmental biology. Annu Rev Cell Dev Biol. 2011:27:213-35.

13. Heinz-Erian P. Unpublished observation. 2010.

14. Dahl M, Tybjaerg-Hansen A, Lange P, Vestbo J, Nordestgaard BG. Change in lung function and morbidity from chronic obstructive pulmonary disease in alpha-1-antitrypsin MZ heterozygotes: a longitudinal study of the general population. Ann Intern Med. 2002;136(4):270-9.

15. Endres W. Inherited metabolic diseases affecting the carrier. J Inherit Metab Dis. 1997:20(1):9-20.

16. Salomon J, Goulet O, Canioni D, Brousse N, Lemale J, Tounian P, Coulomb A, Marinier E, Hugot JP, Ruemmele F, Dufier JL, Roche O, Bodemer C Colomb V, Talbotec C, Lacaille F, Campeotto F, Cerf-Bensussan N, Janecke AR, Mueller T, Koletzko S, Bonnefont JP, Lyonnet S, Munnich A, Poirier F, Smahi A. Genetic characterization of congenital tufting enteropathy: epcam associated phenotype and involvement of SPINT2 in the syndromic form. Hum Genet. 2014;133(3):299-310.

17. Szabo R, Hobson JP, Kristoph K, et al. Regulation of cell surface protease matriptase by HAI2 is essential for placental development, neural tube closure and embryonic survival in mice. Development. 2009:136(15):2653-63.

18. Mitchell KJ, Pinson KI, Kelly OG, et al. Functional analysis of secreted and transmembrane proteins critical to mouse development. Nat Genet. 2001;28(3):241-9.

19. Myerburg MM, Butterworth MB, McKenna EE, et al. Airway surface liquid volume regulates $\mathrm{ENaC}$ by altering the serine protease-protease inhibitor balance: a mechanism for sodium hyperabsorption in cystic fibrosis. J Biol Chem. 2006;281(38):27942-9.

20. Shin D, Gilbert F, Goldstein M, et al. Congenital absence of the vas deferens: incomplete penetrance of cystic fibrosis gene mutations. J Urol. 1997;158(5):1794-8.

\section{Submit your next manuscript to BioMed Central and we will help you at every step:}

- We accept pre-submission inquiries

- Our selector tool helps you to find the most relevant journal

- We provide round the clock customer support

- Convenient online submission

- Thorough peer review

- Inclusion in PubMed and all major indexing services

- Maximum visibility for your research

Submit your manuscript at www.biomedcentral com/submit
Ciomed Central 